\title{
Caractérisation en tant que capteur d'eau de l'enracinement du maïs en parcelle cultivée. I. - Discussion des critères d'étude
}

François TARDIEU \& Hubert MANICHON $\left({ }^{*}\right)$

I.N.R.A., Laboratoire d'Agronomie, I.N.A.-P.G., F 78850 Thiverval-Grignon

(*) I.N.A.-P.G., Chaire d'Agronomie, F 78850 Thiverval-Grignon

\begin{abstract}
Les méthodes de caractérisation du système racinaire en tant que capteur d'eau sont discutées, spécifiquement dans le cas où il existe une forte variabilité horizontale de la densité racinaire liée à la présence d'obstacles structuraux à la pénétration des racines. L'examen des expérimentations et des modèles portant sur le transfert d'eau entre le sol et les racines suggère que, dans ce cas, les conclusions de NEwMAN (1969) ne sont pas valides et que les résistances au transfert de l'eau à l'intérieur du sol peuvent être importantes. La distance entre chaque point du sol et la racine la plus proche reste alors un critère pertinent pour caractériser la résistance au transfert de l'eau entre le sol et les plantes. Des calculs effectués sur des exemples montrent que la fonction de répartition de ces distances dépend fortement de la disposition spatiale des racines. Lorsque celle-ci est de type groupé, un fraction importante du sol se trouve éloignée de la racine la plus proche d'une distance plusieurs fois supérieure à la « demi-distance moyenne entre racines voisines », calculée classiquement en supposant une disposition régulière des racines. Dans ce cas, la caractérisation de l'enracinement par une longueur de racine dans chaque horizon de sol s'avère donc insuffisante ; il est nécessaire d'y inclure l'étude de la disposition spatiale des racines, ce qui n'est pas permis par les techniques actuelles d'observation des systèmes racinaires.
\end{abstract}

Mots clés additionnels : Transferts d'eau sol-plante, disposition spatiale, modèles, état structural.

Characterization of the maize root system under field conditions as a water sink. I. - Criteria for study.

\begin{abstract}
Methods for characterizing root systems as water sinks are discussed, specifically for the case when root density is uneven horizontally, due to the presence of compacted structural elements within the soil profile. A review of published results and models on soil-plant water transfer suggests that NEwmaN's (1969) conclusions are not valid in this case, and that resistances to water transfer within the soil may be appreciable. Accordingly, the distance between each point in the soil and the nearest root remains a pertinent criterion in characterizing soil-plant resistance to water flow. Calculations carried out on examples showed that the cumulative frequency distribution of these distances strongly depended on the spatial arrangement of the roots. If this arrangement followed a clustered pattern, an appreciable proportion of the soil was at a distance from the neighbouring root several times larger than the " mean half distance between neighbouring roots ", classically calculated assuming a regular pattern. In this case, characterization of root systems by the length of roots per layer would prove inadequate, and the spatial arrangement of the roots should also be studied. Classical techniques for observing root systems do not allow this.
\end{abstract}

Additional key words : Soil-plant water transfer, spatial distribution, models, soil structure.

\section{INTRODUCTION}

Il est souvent admis que l'alimentation hydrique du maïs peut être perturbée par l'état physique résultant du travail du sol. Cependant, sauf cas extrême où la profondeur d'enracinement est limitée par un obstacle continu à la pénétration des racines, on connaît assez mal les liaisons entre l'état physique du sol et la capacité du système racinaire à utiliser les réserves en eau.
Nous pensons que ceci est dû, au moins partiellement, au fait que les méthodes classiques de caractérisation du système racinaire (cf. SCHUURMAN \& GOEDEWAGEN, 1965 ; BOHM, 1979) n'ont pas été conçues pour les situations où la colonisation du sol par les racines est perturbée par la présence d'obstacles structuraux à l'enracinement, situations fréquentes en parcelles cultivées (MANichON \& SebillotTe, 1973 ; MANiCHON et al., 1980 ; MEYNARD et al., 1981). Nous nous som- 
mes donc attachés à repenser, spécifiquement pour ces situations, la caractérisation des enracinements en tant que capteurs d'eau.

Le système racinaire est un objet particulièrement difficile à décrire. Si le nombre et le rythme d'émission des racines primaires commencent à être connus (PICARD \& JACQUOT, 1976, sur riz ; KLEPPER et al., 1984, sur blé ; PICARD et al., 1985, sur maïs), la longueur et le nombre des ramifications de celles-ci n'ont encore pu être ni décrits ni modélisés efficacement. Le système que nous cherchons à caractériser ici est donc constitué d'un nombre inconnu de ramifications de plusieurs ordres des racines primaires, de longueurs et de diamètres variables, répartis dans un volume de terre lui-même inconnu.

Par comparaison avec des études effectuées sur plantes isolées situées sur des milieux considérés comme homogènes, la difficulté est encore accrue lors d'études au champ.

- Les racines croissent dans un milieu où la porosité et l'humidité (et donc la résistance mécanique à la pénétration : TAYLOR \& GARDNER, 1963 ; MAERTENS, 1964a) sont spatialement très variables à un instant donné (MANICHON, 1982 ; PAPY, 1984). Ceci est dû en particulier aux discontinuités structurales créées par les outils. Il peut en résulter une répartition irrégulière des racines dans le volume qu'elles colonisent : des différences de densité racinaire entre des volumes de sol contigus, mais d'états structuraux différents, ont ainsi été observés par SEBILLOTTE (1963), EDWARDS et al. (1964), et HENIN et al. (1969). Au champ, la répartition spatiale des racines doit donc être considérée comme inconnue.

- Les racines de chacune des plantes ne peuvent généralement être extraites en totalité du volume de sol dans lequel elles se situent. Il est donc nécessaire d'examiner des volumes élémentaires de sol, afin d'y étudier la densité racinaire (longueur ou poids de racines par unité de volume). L'échantillonnage de ces volumes est rendu délicat par l'existence de l'hétérogénéité évoquée plus haut (de dimension décimétrique), mais aussi par une hétérogénéité métrique, liée à l'état de croissance des pieds étudiés. Les parties aériennes ont, en effet, des masses souvent différentes entre plantes d'un même peuplement végétal (MASLE \& SEBILLOTTE, 1981, sur blé ; DAYNARD \& MULDOON, 1983, sur maïs) ; les travaux qui portent sur l'allométrie de croissance entre les parties aériennes et souterraines (BROUWER, 1966 ; WELBANK et al., 1974) amènent à penser que cette variabilité de la croissance peut se répercuter sur le système racinaire.

Or, pour pouvoir étudier au champ l'utilisation des réserves en eau du sol par les peuplements végétaux, et notamment pour analyser les conséquences de tassements du sol sur cette utilisation, il est nécessaire de pouvoir caractériser les systèmes racinaires en tant que capteurs d'eau. Ce premier article a pour objectif de donner des éléments pour cette caractérisation : dans une $1^{\text {re }}$ partie, nous discuterons des critères pertinents, compte tenu, d'une part, des difficultés évoquées et, d'autre part, des connaissances disponibles sur les mécanismes des transferts d'eau entre le sol et les plantes. Dans une $2^{\mathrm{e}}$ partie, nous confronterons les différentes méthodes existantes au «cahier des charges » retenu.
Un $2^{\mathrm{e}}$ article (TARDIEU \& MANICHON, 1986) présentera une méthode adaptée aux objectifs et aux contraintes de notre étude et la justifiera par de premiers résultats.

\section{CHOIX DE CRITÈRES DE CARACTÉRISATION DE L'ENRACINEMENT}

L'étude de l'enracinement en tant que capteur d'eau a pour but de pouvoir classer, entre plusieurs situations, la résistance au transfert de l'eau entre le sol et les plantes. Cette résistance, définie par VAN DEN HONERT (1948) comme le rapport entre, d'une part, la différence de potentiel hydrique entre le sol et les feuilles et, d'autre part, le flux d'eau à travers les plantes, est classiquement scindée en 3 parties : les résistances au transfert de l'eau dans le sol, à l'interface sol-racines ot entre la surface des racines et les feuilles. Chacune de ces résistances est liée aux caractéristiques des enracinements.

\section{A. Caractérisation du trajet de l'eau à l'intérieur du sol}

\section{Méthode classique : calcul de la distance moyenne entre racines voisines}

Le transfert d'eau a pour moteur la différence de potentiel entre chaque point du sol et la racine la plus proche. Etant donné la géométrie complexe du système racinaire, il est extrêmement difficile d'en étudier le trajet. Plusieurs auteurs ont eu recours à une méthode approchée : ils calculent d'abord la distance moyenne entre racines voisines dans plusieurs horizons du sol et ramènent ensuite le problème à l'étude des transferts radiaux d'eau dans des cylindres de sol verticaux, de volumes égaux et dont le rayon est égal à la demi-distance entre racines voisines, considérée comme la distance maximale entre un point du sol et une racine (GARDNER, 1960 ; COWAN, 1965 ; NEWMAN, 1969 ; MAERTENS et al., 1974 ; TAYLOR \& KLEPPER, 1978).

\section{a) Calcul de la distance moyenne entre racines voisines}

Ce calcul est effectué, pour chaque horizon, à partir de la longueur totale de racines par unité de volume. Il repose implicitement sur l'hypothèse que toute la longueur racinaire est constituée de segments de droite parallèles et verticaux et sur une disposition spatiale hypothétique. Celle-ci est, suivant les auteurs, supposée régulière (sur un plan orthogonal aux droites, les points d'impact sont disposés suivant un réseau régulier) ou aléatoire (les points d'impact sont disposés au hasard, chaque point du plan ayant la même probabilité de recevoir un impact). Les calculs effectués pour plusieurs types de motifs géométriques réguliers et pour une disposition de type aléatoire aboutissent à des résultats proches les uns des autres (tabl. 1).

Sous ces hypothèses, les distances entre points du sol et racines voisines sont alors faibles : le tableau 2 présente les résultats des calculs effectués à partir des données publiées par plusieurs auteurs, dans des situations diverses, pour le maïs à la floraison. Les valeurs obtenues sont, dans presque tous les cas, inférieurs au centimètre, jusqu'à $60 \mathrm{~cm}$ de profondeur. 
TABLEAU 1

Calcul de la distance moyenne entre racines voisines à partir de la longueur de racines par unité de volume (i). Calculation of the average distance between neighbouring roots, from the root length per unit volume (i).

Auteurs

Disposition des racines

Non précisée
Valeur approchée

\begin{abstract}
1) Gardner, 1960 ;
COWAN, 1965 ;

NewMaN, 1969
\end{abstract}

2) MAERTENS et al., 1974

3) TARDIEU, 1984
Régulière, points situés aux sommets de carrés

Régulière, points situés aux sommets de triangles equilatéraus
Formule calculée $1,13(\lambda)^{-1 / 2}$

Aléatoire

$(\lambda)^{-1 / 2}$

$2(\lambda \sqrt{3})^{-1 / 2}$

$1,07(\lambda)^{-1 / 2}$

$(\lambda)^{-1 / 2}$

$(\lambda)^{-1 / 2}$

TABLEAU 2

Valeurs $(\mathrm{mm})$ de la demi-distance moyenne entre racines voisines suivant la profondeur, à la floraison du maïs. (Calculs effectués par la formule 3, tabl. I, à partir des valeurs publiées de la densité racinaire.)

Values $(\mathrm{mm})$ of the half-average distance between neighbouring roots, at maize silking. (Calculations carried out by formula 3, table 1, from published values of root density.)

\begin{tabular}{|c|c|c|c|c|}
\hline \multirow{2}{*}{ Auteurs } & \multicolumn{4}{|c|}{ Profondeurs $(\mathrm{cm})$} \\
\hline & $0-15$ & $15-30$ & $30-45$ & $45-60$ \\
\hline $\begin{array}{l}\text { RoBERTSON et al., } 1980 \\
\text { Traitement irrigué }\end{array}$ & 2,6 & 4,7 & 7,6 & 9,8 \\
\hline $\begin{array}{l}\text { ROBERTSON et al., } 1980 \\
\text { Traitement non irrigué }\end{array}$ & 2,7 & 5,3 & 6,0 & 12,0 \\
\hline Mengel \& BARBER, 1974 & 2,7 & 3,4 & 4,0 & 4,7 \\
\hline $\begin{array}{l}\text { BARBER, } 1971 \\
\text { Traitement labour }\end{array}$ & 4,4 & 4,5 & 4,7 & 4,5 \\
\hline $\begin{array}{l}\text { BARBER, } 1971 \\
\text { Semis direct }\end{array}$ & 3,6 & 4,3 & 6,0 & 5,7 \\
\hline AlLMARAS et al., 1975 & 13,3 & 9,1 & 11,2 & 9,8 \\
\hline
\end{tabular}

b) Effet de la distance à l'absorbant sur la vitesse de dessèchement du sol

L'effet de cette distance a été étudié par plusieurs auteurs (GARDNER, 1960 ; HALLAIRE, 1963 ; COWAN, 1965 ; PASSIOURA \& COWAN, 1968). Ils ont pour cela résolu les équations différentielles obtenues à partir de la loi de DARCY, en posant l'hypothèse que la conductivité hydraulique du sol est constante sur le trajet de l'eau, au cours du temps considéré pour l'étude. En particulier, le modèle de GARDNER d'écoulement d'eau en régime transitoire consiste à calculer, en tout point du cylindre entourant la racine, la chute de potentiel hydrique $\left(\psi-\psi_{0}\right)$ au cours du temps t, nécessaire pour maintenir constant le volume d'eau absorbé par unité de longueur de racines (q : vitesse d'absorption de l'eau en $\mathrm{cm}^{3} . \mathrm{cm}^{-1}$. jour ${ }^{-1}$ )

$$
\psi-\psi_{0}=\frac{\mathrm{q}}{4 \pi \mathrm{k}}\left[\ln \frac{4 \mathrm{Dt}}{\mathrm{a}^{2}}-0,577\right]
$$

où a est la distance entre le point considéré et la racine, $\mathrm{k}$ et $\mathrm{D}$ sont respectivement la conductivité et la diffusivité hydraulique.

Il en ressort qu'entre la périphérie de la racine et les zones plus distantes apparaît une différence de potentiel, d'autant plus grande que l'humidité initiale du sol est plus faible et que la vitesse d'absorption est plus élevée. Les gradients calculés sont d'une dizaine de bars sur $3 \mathrm{~cm}$ en sol initialement sec ( 15 bars) et de quelques dixièmes de bars sur la même distance en sol initialement plus humide ( 5 bars). La conclusion tirée par l'auteur est qu'aucun transfert d'eau appréciable ne peut s'effectuer depuis des points situés à une distance supérieure à quelques $\mathrm{cm}$ de la racine. Les autres modèles cités aboutissent à des conclusions similaires.

Expérimentalement, DUNHAM \& NYE (1973) obtiennent des gradients de potentiel plus marqués que ceux prévus par le modèle GARDNER (ceci pourrait être lié à la baisse de conductivité hydraulique du sol au voisinage de l'absorbant, cf. LANG \& GARDNER, 1970). Après $2 \mathrm{j} 1 / 2$ de dessèchement d'un sol sablo-argileux, initialement à 3 bars, par des racines d'oignon (disposées sur un plan), ces auteurs observent une différence de 15 bars entre le voisinage du plan enraciné et les zones situées à plus de $4 \mathrm{~cm}$ de celui-ci. Au-delà de cette distance, le sol est peu desséché par les racines, même après $6 \mathrm{j}$. Les gradients observés auraient probablement été encore plus marqués pour une texture moins sableuse (GARDNER, 1960 ; PUECH, 1969).

Des gradients importants de potentiel hydrique, conséquence de la résistance au transfert de l'eau, peuvent donc exister à l'intérieur du sol sur des distances de quelques $\mathrm{cm}$, lorsque le sol est encore loin du « point de flétrissement permanent » (16 bars). En revanche, quand les distances sont plus faibles (de l'ordre de celles présentées au tabl. 2), NEWMAN (1969 I et II) considère qu'il ne peut pas exister de différences de potentiel importantes à l'intérieur de la zone enracinée, pour les conductivités hydrauliques et les 
flux habituellement rencontrés au champ. Des calculs fondés sur le modèle de GARDNER, il déduit que la résistance au trajet de l'eau à l'intérieur du sol est négligeable devant celles à l'intérieur de la plante. Ce raisonnement est suivi par d'autres auteurs (REICOSKY \& RITCHIE, 1976 ; WEATHERLEY, 1979 ; EHLERS et al., 1981 ; ZUR et al., 1982). S'il se révélait correct, l'étude de la distance entre racines ne devrait pas être retenue comme critère de caractérisation de l'enracinement.

\section{Discussion : disposition spatiale des racines}

Cependant, ces modes de calculs peuvent fortement sous-estimer l'ordre de grandeur des différences de potentiel à l'intérieur du sol. D'une part, ils négligent la baisse de conductivité hydraulique à proximité des racines, mise en évidence par LANG \& GARDNER (1970, cf. paragraphe précédent), d'autre part, ils sont fondés sur 2 hypothèses, à notre avis peu réalistes dans les conditions du champ, qui toutes deux tendent à sous-estimer les résistances à l'intérieur du sol :

- La vitesse d'absorption de l'unité de longueur de racine est supposée uniforme sur l'ensemble du système racinaire. Or, la densité racinaire diminuant avec la profondeur, les premiers horizons du sol sont desséchés les premiers ; ceci entraîne une diminution de la vitesse d'absorption (par unité de longueur) des racines qui s'y trouvent. Le maintien de la transpiration de la plante implique alors une vitesse d'absorption des racines situées en profondeur considérablement plus élevée que la vitesse moyenne calculée sur la longueur totale de racines (GARDNER, 1964 ; MAERTENS et al., 1974 ; TAYLOR \& KLEPPER, 1978).

- A l'intérieur de chaque horizon, les valeurs des distances prises en compte dans les calculs sont fondées sur les hypothèses de verticalité et de régularité de la disposition des racines.

D'une part, ces dernières hypothèses sont contradictoires avec le fait que le système racinaire est ramifié. Au vu de nos propres observations, s'il semble raisonnable de considérer que les racines primaires sont approximativement verticales au-delà de la couche labourée (dans la mesure où elles empruntent les galeries de vers de terre et les fentes de retrait, surtout verticales), les ramifications de ces dernières, de quelques $\mathrm{cm}$ de longueur, sont en revanche sub-horizontales, ce qui a pour conséquence une différence de densité racinaire entre zones proches et éloignées des racines primaires.

D'autre part, lorsqu'il existe des obstacles structuraux non pénétrés par les racines, certains volumes de sol sont plus colonisés que d'autres (cf. Introduction). Dans ce cas, il peut exister des distances de l'ordre de plusieurs cm (dimension des obstacles) entre points du sol et la racine la plus proche, même si la distance moyenne entre racines est faible.

Si de telles distances entraînent une baisse de la disponibilité de l'eau du sol (cf. § 1.), il s'ensuit une variabilité spatiale de l'absorption d'eau à l'intérieur de la zone enracinée, ce qui serait cohérent avec les mesures de VALANCOGNE \& DAUDET (1977), qui ont observé au champ une forte variabilité horizontale du potentiel hydrique dans le sol sous une culture de maïs. Celle-ci ne peut résulter que de l'existence d'une forte résistance au transfert de l'eau à l'intérieur du sol. Une simulation de BALDWIN et al. (1972), réalisée à l'aide d'un analogue électrique, suggère également que l'absorption d'eau par un système racinaire est d'autant plus faible que la disposition des racines s'éloigne du type régulier. L'étude de cette disposition et un autre type de calcul des distances entre les points du sol et la racine la plus proche semblent donc nécessaires à la caractérisation du trajet de l'eau entre le sol et les racines.

\section{Méthodes d'étude de la disposition des racines et conséquences pour le calcul des distances}

a) Les techniques statistiques actuellement disponibles ne permettent d'effectuer cette étude qu'en 2 dimensions. La seule possibilité est donc d'analyser la disposition des impacts de racines sur des plans que nous choisirons horizontaux afin qu'ils soient orthogonaux à la direction privilégiée des racines.

En plus des dispositions de type régulier et de type aléatoire, ROGERS (1974) définit un type groupé pour lequel, sur le plan étudié, un point d'impact a d'autant plus de chance de se trouver à une position donnée que celle-ci est plus proche d'autres impacts. Il y a alors « autocorrélation spatiale » (CLIFF \& ORD, 1973). L'étude des types de dispositions peut être menée par plusieurs méthodes qui, après un maillage du plan, permettent de tester l'hypothèse selon laquelle la disposition considérée diffèrerait significativement d'une disposition de type aléatoire, pour le maillage considéré : les résultats de ces tests dépendent, en effet, de celui-ci, la maille de l'hétérogénéité pouvant être définie comme la maille d'étude à partir de laquelle une disposition étudiée cesse d'être groupée. Deux méthodes sont particulièrement adaptées à l'étude de l'enracinement :

- La méthode des autocorrélations spatiales (MORAN, 1948, cité par CLIFF \& ORD, 1973), fondée sur l'étude des contacts entre les cases où se situent des impacts ( $"$ Noires ») et les cases vides ( Blanches $\gg)$. Trois types de contacts peuvent donc être définis : «Noir-Noir », "Noir-Blanc» et "BlancBlanc ». La méthode consiste à comparer le nombre de contacts de chaque type avec celui qui serait observé si la disposition des impacts était aléatoire (les contacts «Noir-Noir » et «Blanc-Blanc» sont d'autant plus nombreux que la disposition est plus groupée). Pour l'étude de la variabilité des distances entre racines, elle convient particulièrement à des maillages de petite dimension ( 1 à $2 \mathrm{~cm}$ ) et permet ainsi d'étudier les hétérogénéités de maille centimétrique.

- La méthode des quadrats («Quadrat method», GRIEG SMITH, 1964 ; ROGERS, 1974), qui consiste à compter le nombre d'impacts à l'intérieur de chaque case. GRIEG SMITH montre que, si la disposition est aléatoire, ce nombre suit une loi de PoIsSON; son espérance et sa variance sont donc égales. Le test consiste à étudier le rapport de ces 2 variables, qui est d'autant plus différent de 1 que la disposition est plus éloignée d'une disposition aléatoire. Cette méthode ne peut être utilisée que pour des cases de dimensions relativement grandes par rapport au diamètre des raci- 
nes et est donc adaptée à la recherche d'hétérogénéités de maille décimétrique.

Les tableaux 3 et 4 présentent les résultats de ces tests pour 3 dispositions typiques offertes à titre d'exemple à la figure 1 (pour les modalités de calculs, voir TARDIEU, 1984). Il apparaît que les 3 dispositions présentées appartiennent respectivement aux types régulier, aléatoire et groupé, pour les 2 mailles étudiées.

b) Lorsque la disposition des impacts est groupée, la distance moyenne entre racines calculée par les formules présentées au tableaı 2 cesse d'être égale à la distance moyenne vraie entre racines voisines, qui est d'autant plus faible que le groupement est plus prononcé (ROGERS, 1974). En revanche, une proportion importante de points du plan se trouve à une distance d'un impact très supérieure à la demi-distance moyenne. Il existe alors une très forte variabilité des distances entre points du plan et impact le plus proche.

Afin d'étudier les conséquences du type de disposition sur cette variabilité, nous avons calculé la fonction de répartition des distances entre chaque point du plan et l'impact le plus proche, pour les 3 cartes présentées à la figure 1. Après quadrillage de celles-ci (maille de $1 \mathrm{~cm}$ si leurs dimensions sont de $80 \times 30 \mathrm{~cm}$ ), nous avons calculé, pour chaque case de la carte considérée, la distance euclidienne entre le centre de gravité de cette case et le centre de gravité de la case la plus proche où se trouve un impact. La fonction de répartition des distances est ensuite calculée à partir de la fréquence des distances ainsi obtenues. Les résultats de cette analyse montrent (fig. 2) que, si pour la disposition régulière, 90 p. 100 des distances sont inférieures à la «demi-distance moyenne » entre les
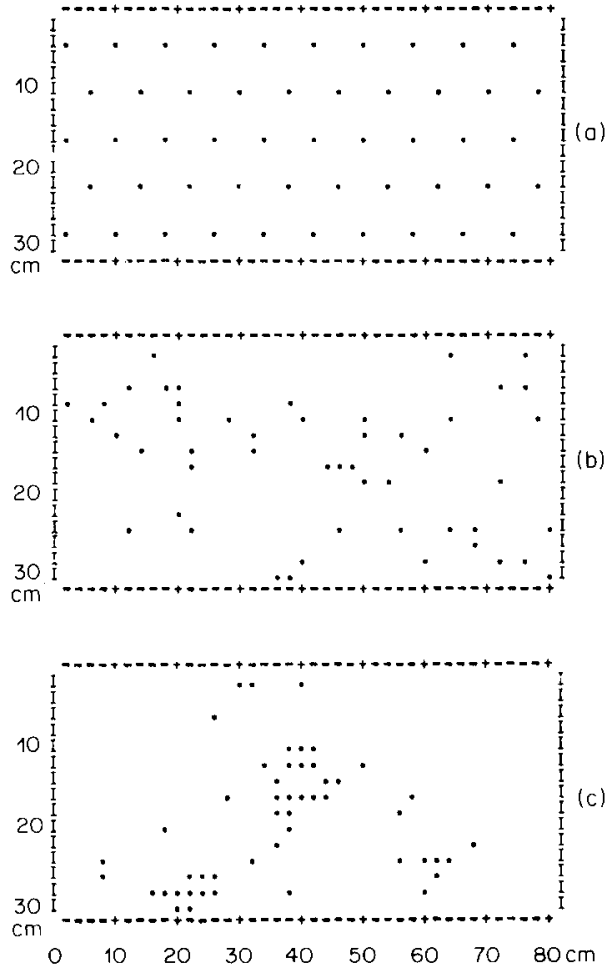

Figure 1

Exemples de dispositions des impacts de 50 racines sur un plan. (a) régulier; (b) aléatoire; (c) groupé.

Examples of the pattern of 50 roots impacting on a plane. (a) regular ; (b) random; (c) clustered.

points d'impact (calculée à partir de la formule 3 présentée au tableau 1), cette proportion n'est que de 54 p. 100 pour la disposition aléatoire et de 35 p. 100 pour la disposition groupée présentée. Pour cette der-

TABLEAU 3

Résultats du test d'autocorrélation spatiale (maille : $2 \mathrm{~cm}$ ) pour les 3 cartes présentées à la figure 1 .

Valeur $d u$ « $t$ » de Student dans la comparaison entre nombre de contacts observés et calculés sous l'hypothèse d'une disposition aléatoire. Results of the spatial autocorrelation tesi for the three maps presented in figure 1.

Student's " $t$ " values, for the difference between observed number of contacts and number calculated assuming random distribution.

\begin{tabular}{|c|c|c|c|c|}
\hline $\begin{array}{l}\text { Carte } \\
\text { étudiée }\end{array}$ & Noir-Noir & $\begin{array}{l}\text { Contacts } \\
\text { Noir-Blane }\end{array}$ & Blanc-Blanc & $\begin{array}{c}\text { Conclusion } \\
\text { Type de disposition }\end{array}$ \\
\hline$a$ & $-4,2^{* *}$ & $2,0^{*}$ & $-4,1^{* *}$ & Régulière \\
\hline$b$ & 0,21 (NS) & 0,0 (NS) & 0,1 (NS) & Aléatoire \\
\hline$c$ & $13,4^{* *}$ & $-3,4^{* *}$ & $4,5^{* *}$ & Groupée \\
\hline
\end{tabular}

\section{TABLEAU 4}

Résultats du test des quadrats (maille: $10 \mathrm{~cm}$ ) pour les 3 cartes présentées à la figure 1 : comparaison à une disposition aléatoire. Result of the quadrat test method for the three maps presented in figure $1:$ departure from a random pattern.

\begin{tabular}{|c|c|c|c|c|}
\hline \multirow[b]{2}{*}{$\begin{array}{l}\text { Carte } \\
\text { étudiée }\end{array}$} & \multicolumn{2}{|c|}{ Nombre d'impacts/Case } & \multirow[b]{2}{*}{ «t»calculé } & \multirow[b]{2}{*}{$\begin{array}{c}\text { Conclusion } \\
\text { Type de disposition }\end{array}$} \\
\hline & Moyenne & $\frac{\text { Variance }}{\text { Moyenne }}$ & & \\
\hline$a$ & 2,08 & 0,29 & $-2,4 * *$ & Régulière \\
\hline$b$ & 2,08 & 1,16 & 0,56 (NS) & Aléatoire \\
\hline$c$ & 2,08 & 2,38 & $8,06^{* *}$ & Groupée \\
\hline
\end{tabular}




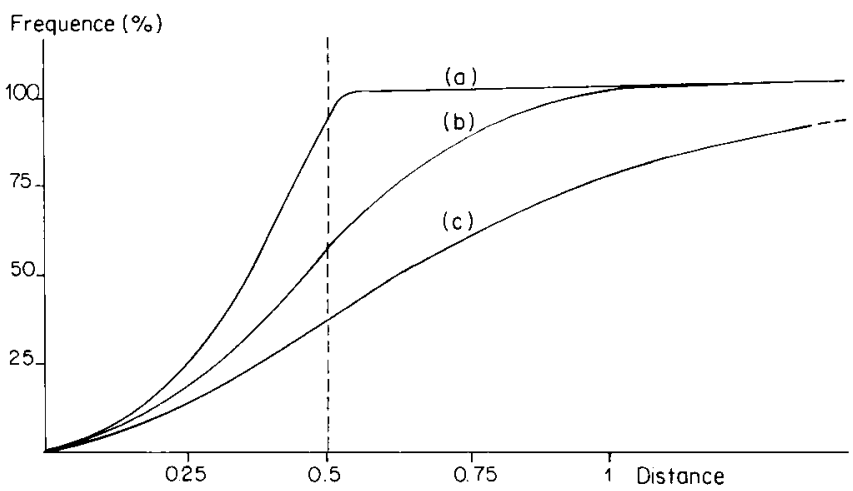

Figure 2

Fonction de répartition des distances entre points du plan et racine la plus proche, pour chaque carte de la figure I. Distances exprimées en fraction de la "distance moyenne entre racines voisines" calculée par la formule 3, tableau 1.

Cumulative frequency distribution of distances between points of the plane and the neighbouring root. Distances in fractions of the "average distance between neighbouring roots", calculated by formula 3, table 1 .

nière, près d'un quart du plan est, en revanche, situé à une distance égale ou supérieure à 2 fois la distance maximale calculée dans les modèles classiques.

Lorsqu'il existe dans le sol des obstacles à la pénétration des racines, entraînant une disposition groupée de celles-ci, le simple calcul de la " distance moyenne entre racines » est donc insuffisant pour caractériser la résistance au trajet de l'eau à l'intérieur du sol. Il est nécessaire d'y ajouter soit une caractérisation du type de disposition des impacts de racines sur des plans horizontaux, soit un calcul direct de la fonction de répartition des distances entre chaque point du plan et l'impact le plus proche.

\section{B. Prise en compte des résistances au transfert de l'eau à l'interface sol-racines et entre la surface des racines et les feuilles}

Ces 2 résistances, ainsi que la comparaison de leurs ordres de grandeur respectifs avec les résistances à l'intérieur du sol, ont fait l'objet d'un grand nombre de publications dont les résultats sont souvent contradictoires. Nous ne chercherons pas à les synthétiser ici (cf. les articles de NEWMAN, 1969, 1976 ; BLIZZARD \& BOYER, 1980 ; MOLZ, 1981), mais à en extraire les aspects concernant la caractérisation de l'enracinement : est-il nécessaire de distinguer plusieurs fractions à l'intérieur du système racinaire au moment de la caractérisation de celui-ci ?

\section{Interface sol-racines}

L'observation des racines au champ montre qu'une fraction importante de leur surface n'est pas en contact avec la terre ; par ailleurs, leur diamètre se réduit lorsque le potentiel hydrique des plantes diminue (HUCK et al., 1970), créant ainsi une discontinuité à l'interface sol-racines. Ces défauts de contact pourraient être à l'origine d'une résistance au transfert de l'eau (WEATHERLEY, 1979). TINKER (1976) discute l'ordre de grandeur de celle-ci : lorsque les lames d'air sont de faible épaisseur (de l'ordre du $1 / 10 \mathrm{~mm}$ ), il estime que cette résistance est faible à cause, d'une part, des transferts à travers les poils racinaires et les zones de contact sol-racines qui subsistent, d'autre part, des transferts en phase gazeuse (CowAN \& MILTHORPE, 1968). Ce raisonnement ne s'applique pas, en revanche, aux racines séparées de la terre par plusieurs $\mathrm{mm}$ (racines de morphologie typique avec des poils racinaires surdéveloppés, situées au sein de cavités entre mottes tassées ou entre bandes de labour). Celles-ci ont probablement une absorption très limitée, il nous a paru nécessaire de les distinguer.

\section{Résistance entre la surface des racines et les feuilles}

Les auteurs qui ont étudié l'absorption d'une racine reliée à une plante en transpiration [HAYWARD et al. (1942) cités par NEWMAN (1976) et MAERTENS (1971) sur maïs ; BROUWER (1953) et RowSE \& GOODMAN (1981) sur Vicia faba; GRAHAM et al. (1974) sur Cucurbita pepo] ont montré que, en l'absence d'autre résistance que les résistances internes à la plante (racines placées en solution nutritive), toutes les parties du système racinaire (indépendamment de l'âge ou de l'ordre de ramification) sont potentiellement capables d'absorber un flux d'eau nettement plus grand que ceux observés au champ. La comparaison de la perméabilité des parties distales et apicales de la racine fournit des résultats différents suivant les auteurs, BROUWER (1953) montrant que le classement entre ces 2 parties n'est pas stable lorsque la transpiration de la plante varie. Par ailleurs, ROWSE \& GOODMAN (1981) n'ont pas observé de différence de vitesse d'absorption par unité de longueur du pivot et de racine secondaire d'une plante de féverole.

A une autre échelle, TAYLOR \& KLEPPER (1973, 1975) montrent que, en sol humide (les résistances à l'intérieur du sol et à l'interface sol-racine sont alors très faibles), l'absorption d'eau par unité de longueur de racine est semblable sur tout le système racinaire. Ce résultat ne paraît pas être modifié après un cycle dessication-humectation de l'environnement des racines : DIRKSEN \& RAATS (1985) ont montré, sur luzerne, qu'après réhumectation de couches superficielles préalablement desséchées jusqu'à la moitié de la capacité de rétention, les racines qui y sont situées recommencent immédiatement à absorber l'eau à une vitesse inchangée.

Ces expériences tendent à montrer que, lorsque l'environnement est favorable, la totalité du système racinaire peut être considérée comme potentiellement fonctionnelle. Dans l'état actuel des connaissances, il ne paraît donc pas déraisonnable de ne distinguer ni les ordres de ramification des racines, ni l'âge de celles-ci, pour caractériser le système racinaire; nous n'aurions pas, en revanche, de base claire pour distinguer les racines sur des critères morphologiques, suivant leur capacité à absorber l'eau.

\section{III. « CAHIER DES CHARGES »ET ADÉQUATION DES MÉTHODES EXISTANTES}

A partir des paragraphes précédents, il est possible de définir un « cahier des charges » pour la caractérisation en tant que capteurs d'eau de systèmes racinaires dans des sols d'état structural défavorable. 
- La méthode choisie doit permettre d'évaluer la fonction de répartition des distances entre les points du sol et les racines ou, au minimum, de pouvoir classer sur ce critère les systèmes racinaires observés. $\mathrm{Au}$ vu de ce qui précède, ceci est la condition la plus importante.

- Elle doit permettre de distinguer les racines situées dans des cavités structurales de celles en contact avec la terre.

- Enfin, elle doit prendre en compte l'hétérogénéité métrique du système racinaire, liée à la diversité des états de croissance des plantes, afin de pouvoir obtenir une évaluation à l'échelle du peuplement végétal. Il est donc nécessaire de pouvoir effectuer plusieurs répétitions. La méthode retenue doit donc être relativement rapide.

Face à ces exigences, on peut classer en 3 groupes les techniques d'étude de l'enracinement au champ : les techniques fondées sur le prélèvement d'échantillons, l'observation en continu à travers un tube transparent et les techniques qui peuvent aboutir à une cartographie de la densité racinaire.

\section{A. Le prélèvement d'échantillons de terre}

La technique la plus utilisée consiste à prélever des carottes verticales de sol, découpées ensuite en horizons où la longueur de racines par unité de volume est mesurée, après lavage de la terre (cf. SCHUURMAN \& GOEDEWAGEN, 1965). C'est la technique adoptée, en particulier, par les auteurs dont les résultats figurent au tableau 2. D'autres technique reposent sur un échantillonnage de la face verticale d'une fosse: MAERTENS (1964b), MAERTENS et al. (1974) et CHOPART (1980) y prélèvent une série de carottes horizontales à plusieurs profondeurs.

La variable de sortie est ici la densité moyenne de racines par horizon. Les auteurs qui étudient des cultures à faible densité de peuplement y associent, en général, une covariable distance au rang. En revanche, il est plus rare qu'un indicateur de la variabilité de la densité racinaire soit présentée en même temps que la densité moyenne.

Si l'échantillonnage est correctement effectué, ce qui nécessite de prendre en compte les hétérogénéités décimétrique et métrique de la densité racinaire, une estimation non biaisée de la longueur de racines par horizon peut être obtenue par cette méthode. Par contre, celle-ci ne fournit aucune donnée sur la disposition spatiale des racines : elle est, en général, associée à la méthode de calcul des « distances moyennes entre racines voisines ", exposée au $\S$ II A.1. Par ailleurs, elle ne donne pas d'indications sur la nature du contact entre la terre et les racines.

\section{B. Observations en continu à travers un tube trans- parent}

Cette technique est développée actuellement par MAERTENS \& ClAUZEL (1982) et PiCARD (travaux en cours). Elle est la seule à permettre de suivre en continu la progression du front racinaire au cours du temps. Elle peut également être utilisée pour évaluer la densité racinaire, si l'interface entre le tube et le sol n'est pas perturbée par des tassements ou un retrait volumique de la terre ; mais elle ne permet pas d'étudier la disposition spatiale des racines et n'est donc pas suffisante, à elle seule, pour satisfaire aux exigences présentées plus haut.

\section{Techniques aboutissant à une cartographie}

- La $1^{\text {re }}$ de celles-ci consiste à prélever une tranche verticale de sol, généralement après avoir enfoncé une planche à clous sur la face verticale d'un profil (WEAVER, 1926 ; FOTH, 1962 ; NELSON \& ALlMARAS, 1969). Après lavage de la terre, le système racinaire peut être observé sans que les racines primaires aient été fortement déplacées. Cette technique a été surtout utilisée pour étudier les caractéristiques morphologiques des enracinements (longueur, direction et ramifications des différents ordres de racines, notamment). D'autres auteurs l'ont cependant adoptée pour évaluer la longueur de racines par horizon, puis la distance moyenne entre racines (AllMARAS et al., 1975). L'information recueillie sur disposition spatiale des racines n'est alors pas utilisée.

Cette méthode qui permet d'évaluer précisément la longueur racinaire et (potentiellement) d'en étudier la répartition spatiale est extrêmement lourde. Les auteurs qui l'ont utilisée n'ont généralement pu ni étudier toute la profondeur enracinée, ni effectuer de répétitions. Elle ne donne, par ailleurs, pas d'indications sur le contact terre-racines.

- La méthode de cartographie in situ consiste à quadriller (maille de $5 \mathrm{~cm}$ en général) un plan vertical coupant le volume enraciné et à apprécier visuellement la densité racinaire dans chacune des cases ainsi définies. Elle a été adoptée par MANICHON (1982) pour l'étude des conséquences de l'état structural du sol sur la pénétration des racines. ВоHм (1976), ВоHм et al. (1977) et EHLERS et al. (1981) l'utilisent pour estimer la longueur totale de racines par horizon, d'où ils déduisent la distance moyenne entre racines voisines dans chacun de ceux-ci. Les données disponibles sur l'hétérogénéité horizontale de la densité racinaire ne sont alors pas utilisées pour le calcul des distances (bien que cette hétérogénéité soit très prononcée dans les cartes publiées par cet auteur).

Cette méthode, moins précise que les autres pour l'évaluation de la longueur racinaire (ВоHM et al., 1977), pourrait, par contre, permettre une étude fine de la disposition spatiale des racines, à condition de choisir une maille d'étude plus petite que celle utilisée par les auteurs cités et de ne pas limiter l'étude à la cartographie d'un plan vertical (cf. \& II A.3.). Elle permet également l'appréciation in situ du contact terre-racines.

\section{CONCLUSION}

A part la cartographie in situ, les méthodes actuelles d'étude de l'enracinement au champ peuvent fournir des évaluations relativement précises de la longueur de racines par horizon. En l'état actuel, elles ne permettent pas, en revanche, d'étudier la disposition des racines. Pour le calcul des distances entre celles-ci, 
elles sont donc associées aux hypothèses, très fortes, de disposition des racines discutées au $\S$ II A.2.

$\mathrm{Si}$ des distances de l'ordre de quelques $\mathrm{cm}$ entre points du sol et racine la plus proche provoquent des résistances importantes au transfert de l'eau (ce qui est fortement suggéré par les connaissances actuelles, mais encore discuté), il est nécessaire de prendre en compte la disposition des racines pour étudier le système racinaire. On pourrait alors substituer à l'étude de la «distance moyenne entre racines voisines ", celle de la fonction de répartition des distances entre les points du sol et la racine la plus proche.

Ce type d'étude est particulièrement nécessaire à la caractérisation de systèmes racinaires qui se développent dans des sols où la résistance à la pénétration est spatialement variable (présence de mottes tassées ou de traces de roues discontinues dans la couche labourée, fissurations de celle-ci ou des couches sousjacentes...). Il l'est à plus forte raison si l'objectif poursuivi est de comparer des systèmes racinaires dans des sols d'états structuraux différents : il est vraisemblable que la caractérisation du système racinaire par une distance moyenne entre racines arriverait, dans ces cas, à des erreurs d'interprétation dans l'étude de l'utilisation des réserves en eau du sol par le peuplement végétal.

Reçu te 18 février 1985. Accepté le 27 novembre 1985.

\section{RÉFÉRENCES BIBLIOGRAPHIQUES}

Allmaras R. R., Nelson W. W., Voorhees W. B., 1975. Soybean and corn rooting in southwestern Minnesota. II : Root distributions and related water inflow. Soil Sci. Soc. Am. Proc., 39, 771-777.

Baldwin J. P., Tinker P. B., Nye P. H., 1972. Uptake of solutes by multiple root systems from soil. II : The theoretical effects of rooting density and pattern on uptake of nutrients from soil. Plant and Soil, 36, 693-708.

Barber S. A., 1971. Effect of tillage practice on corn root distribution and morphology. Agron. J., 63, 724-726.

Blizzard W. E., Boyer J. S., 1980. Comparative resistance of the soil and the plant to water transport. Plant Physiol., 66, 809-814.

Bohm W., 1976. In situ estimation of rool length at natural soil profiles. J. Agric. Sci. Camb., 69, 415-418.

Bohm W., 1979. Methods of studying root systems, Berlin, $188 \mathrm{p}$. Bohm W., Maduakor H., Taylor H. M., 1977. Comparison of five methods for characterizing soybean rooting density and development. Agron. J., 69, 415-419.

Brouwer R., 1953. Water absorption by roots of Vicia faba at various transpiration strength. I : Analysis of the uptake and the factors determining it. Proc. Kongr. Ned. Acad. Wetensch, 56, 106115 .

Brouwer R., 1966. Root growth of grasses and cereals, 153-156. In Milthorpe F. L. \& Ivins J. D. : "The growth of cereals and grasses », Butterworth, London, $358 \mathrm{p}$.

Chopart J. L., 1980. Étude au champ des systèmes racinaires des principales cultures pluviales au Sénégal. Thèse Doct. Ing., I.N.P. Toulouse, $160 \mathrm{p}$.

Cliff A. D., Ord J. K., 1973. Spatial autocorrelation. Pion Ltd., London, $178 \mathrm{p}$.

Cowan I. R., 1965. Transport of water in the soil-plant atmosphere system. J. Appl. Ecol., 2, 221-239.

Cowan I. R., Milthorpe F. L., 1968. Plant factors influencing the water status of plant tissue, 137-193. In Kozlowski T. T. : «Water deficits and plant growth ». Acad. Press, London, $390 \mathrm{p}$.

Daynard T. B., Muldoon J. F., 1983. Plant to plant variability of maize plants grown at different densities. Can. J. Plant. Sci., 63, 45-59.

Dirksen C., Raats P. A. C., 1985. Water uptake and release by alfalfa roots. Agron. J., 77, 621-626.

Dunham R. J., Nye P. H., 1973. The influence of water content on the uptake of ions by roots. I : Soil water content gradient near a plane of onion roots. J. Appl. Ecol., 10, 585-598.

Edwards W. M., Fehrenbacher J. B., Vavra J. P., 1964. The effect of discrete ped density on corn root penetration in a planosoil. Soil Sci. Soc. Am. Proc., 28, 560-564.

Ehlers W., Grimme K., Baeumer K., Stulpnagel R., Kopke U., Bohm W., 1981. Flow resistance in soil and plant during field growth of oats. Geoderma, 25, 1-12.

Foth H. D., 1962. Root and top growth of corn. Agron. J., 54, 4952.
Gardner W. R., 1960. Dynamic aspects of water availability to plants. Soil Sci., 89, 63-73.

Gardner W. R., 1964. Relation of root distribution to water uptake and availability. Agron. J., 56, 35-41.

Grieg Smith P., 1964. Quantitative plant ecology. 2nd edition, Butterworths, London, $256 \mathrm{p}$.

Graham J., Clarkson D. T., Sanderson J., 1974. Physiology of root function. A.R.C. Letcombe Lab. Annu. Rep. for 1973, 9-12.

Hallaire M., 1963. Le potentiel efficace de l'eau dans le sol en régime de dessèchement. Ann. Agron., 14, 393-428.

Henin S., Gras R., Monnier G., 1969. Le Profil Cultural. Masson, Paris, $332 \mathrm{p}$.

Huck M. G., Klepper B., Taylor H. M., 1970. Diurnal variations in

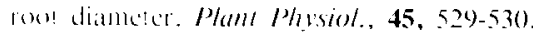

Klepper B., Belford R. K., Rickman R. W., 1984. Root and shoot development in winter wheat. Agron. J., 76, 117-122.

Lang A. R. G., Gardner W. R., 1970. Limitation to water flow from soil to plants. Agron. J., 62, 693-694.

Maertens C., 1964a. Influence des propriétés physiques des sols sur le développement radiculaire et conséquences sur l'alimentation hydrique et azotée des cultures. Sci. Sol, 2, 31-41.

Maertens C., 1964b. La résistance mécanique des sols à la pénétration : ses facteurs et son influence sur l'enracinement. Ann. Agron., $15,539-554$.

Maertens C., 1971. Etude expérimentale de l'alimentation minérale et hydrique du mais. $b$ ) Capacité d'absorption des parties basales et ipricalen de racines de Zea mars. C.R. Acat. Sai. Paris, 273, 730732.

Maertens C., Clauzel Y., 1982. Premières observations sur l'utilisation de l'endoscopie dans l'étude de l'enracinement in situ de plantes cultivées. Agronomie, 2, 677-680.

Maertens C., Blanchet R., Puech J., 1974. Influence de différents régimes hydriques sur l'absorption de l'eau et des éléments minéraux par les cultures. I - Régimes hydriques, systèmes racinaires et modalités d'alimentation en eau. Ann. Agron., 25, 575-586.

Manichon H., 1982. Influence des systèmes de culture sur le profil cultural: Elaboration d'une méthode de diagnostic basée sur l'observation morphologique. Thèse Doct. Ing. I.N.A.-P.G., Paris, $214 \mathrm{p}$.

Manichon H., Sebillotte M., 1973. Etude de la monoculture du mais. Résultats d'enquêtes agronomiques en Béarn. Doc. Ronéo., Chaire d'Agronomie, I.N.A.-P.G., Paris, 108 p.

Manichon H., Ribeyre Cl., Rollier M., 1980. Relations entre l'état physique du sol et la nutrition minérale du tournesol. IX Conf. Int. del Girasol, II, 166-173.

Masle-Meynard Josette, Sébillotte M., 1981. Etude de l'hétérogénéité d'un peuplement de blé d'hiver. I : Notion de structure du peuplement. Agronomie, 1 (3), 207-216.

Mengel D. B., Barber S. A., 1974. Development and distribution of the corn root system under field conditions. Agron. J., 66, 341-344. 
Meynard J. M., Boiffin J., Caneill J., Sébillotte M., 1981 . Elaboration du rendement et fertilisation azotée du blé d'hiver. II : Types de réponse à la fumure azotée et application de la méthode du bilan prévisionnel. Agronomie, 1 (9), 795-806.

Molz F. J., 1981. Models of water transport in the soil-plant system : a review. Water Resour. Res., 61, 751-755.

Nelson W. W., Allmaras R. R., 1969. An improved monolith method for excavating and describing roots. Agron. J., 61, 751-755.

Newman E. I., 1969. Resistance to water flow in soil and plant. I Soil resistance in relation to amounts of root : theoretical estimates. J. Appl. Ecol., 6, 1-2. II - A review of experimental evidence on the rhizosphere resistance. J. Appl. Ecol., 6, 261-272.

Newman E. I., 1976. Water movement through root systems. Phil. Trans. R. Soc. Lond. B., 273, 463-478.

Papy F., 1984. Comportement du sol sous l'action des façons de reprise d'un labour au printemps. Effet des conditions climatiques et de l'état structural. Thèse Doct. Ing. I.N.A.-P.G., Paris, 232 p.

Passioura J. B., Cowan I. R., 1968. On solving the non-linear diffusion equation for the radial flow of water to roots. Agr. Meteorol., 5, 129-134.

Picard D., Jacquot M., 1976. Rythmes d'émission comparés des racines nodales de trois variétés de riz. Agron. Trop., 31, 159-169.

Picard D., Jordan M. O., Trandel R., 1985. Rythme d'apparition des racines primaires du maïs (Zea mays L.). I : Etude détaillée pour une variété en un lieu donné. Agronomie, 5 (8), 667-676.

Puech T., 1969. Etude expérimentale de la circulation d'eau non saturante de différents sols vers une zone d'absorption. Ann. Agron., 20, 245-261.

Reicosky D. C., Ritchie J. T., 1976. Relative importance of soil resistance and plant resistance in root water absorption. Soil Sci. Soc. Am. J., 40, 293-297.

Robertson W. K., Hammond L. C., Johnson J. T., Boote K. J., 1980. Effect of plant-water stress on root distribution of corn, soybeans and peanuts in sandy soil. Agron. J., 72, 548-550.

Rogers A., 1974. Statistical analysis of spatial distribution. The quadrat method. Pion Ltd., London, $164 \mathrm{p}$.

Rowse H. R., Goodman D., 1981. Axial resistance to water movement in broad bean (Vicia faba) roots. J. Exp. Bot., 32, 591-598.

Schuurman D. T., Goedewagen M. A. J., 1965. Methods for examination of root systems and roots. Center for Agric. publ. and doc. ed. Wageningen, $86 \mathrm{p}$.
Sébillotte M., 1963. Action de la structure du sol sur une culture de féverole. C.R. Acad. Agric. Fr., 711-716.

Tardieu F., 1984. Etude au champ de l'enracinement du mais. Influence de l'état structural sur la répartition des racines. Conséquences sur l'alimentation hydrique. Thèse Doct. Ing., Paris, 232 p.

Tardieu F., Manichon H., 1986. Caractéristique en tant que capteur d'eau de l'enracinement du maîs. II. Une méthode d'étude de la répartition verticale et horizontale des racines. Agronomie, 6 (5).

Taylor H. M., Gardner H. R., 1963. Penetration of cotton seedling taproots as influenced by bulk density, moisture content and strength of soil. Soil Sci., 96, 153-156.

Taylor H. M., Klepper B., 1973. Rooting density and water extraction patterns for corn. Agron. J., 65, 965-968.

Taylor H. M., Klepper B., 1975. Water uptake by cotton root systems : an examination of assumptions in the single root model. Soil Sci., 120, 57-67.

Taylor H. M., Klepper B., 1978. The role of rooting characteristics in the supply of water to plants. Adv. Agron., 30, 99-128.

Tinker P. B., 1976. Roots and water. Transport of water to plant roots in soil. Phil. Trans. R. Soc. Lond. B., 445-461.

Valancogne C., Daudet F., 1977. Etude in situ du potentiel hydrique sous une culture de maïs à l'aide d'un système automatique de mesures à micro-psychomètre à effet Peltier. Ann. Agron., 28, 137157.

Van den Honert T. H., 1948. Water transport in plants as a catenary process. Discuss. Faraday Soc., 3, 146-153.

Weatherley P. E., 1979. The hydraulic resistance of the soil root interface. A cause of water stress in plants, 275-286. In Harley J. L. \& Scott Russel R. : "The soil root interface». Academic Press, New York, 448 p.

Weaver J. E., 1926. Root development of field crops. Mc GrawHill, New York, $291 \mathrm{p}$.

Welbank P. J., Gibb M. J., Taylor P. J., Williams E. D., 1974. Root growth of cereal crops. Rep. Rothamsted Exp. Sin. for 1973, part 2, 22-26.

Zur B., Jones J. W., Boote K. J., Hammond L. C., 1982. Total resistance to water flow in field soybeans. II : Limiting soil moisture. Agron. J., 74, 99-105. 